\title{
1 Effects of graphene oxide and graphite on soil bacterial and fungal
}

\section{2 diversity}

4 Christian Forstner ${ }^{\mathrm{a}}$, Thomas G. Orton ${ }^{\mathrm{b}}$, Adam Skarshewski ${ }^{\mathrm{a}}$, Peng Wang ${ }^{\mathrm{b}, 1}$, Peter M. Kopittke ${ }^{\mathrm{b}}$,

5 Paul G. Dennis ${ }^{\mathrm{a} *}$

6

$7 \quad{ }^{a}$ School of Earth and Environmental Sciences, The University of Queensland, St Lucia, Brisbane,

8 QLD 4072, Australia

9 bshool of Agriculture and Food Sciences, The University of Queensland, St Lucia, Brisbane,

10 QLD 4072, Australia

11

12 Running Head: Graphene oxide affects soil microbial diversity

13

14 *Address correspondence to Paul G. Dennis, p.dennis@uq.edu.au

15

$16{ }^{\text {l} P r e s e n t ~ a d d r e s s: ~ C o l l e g e ~ o f ~ R e s o u r c e s ~ a n d ~ E n v i r o n m e n t a l ~ S c i e n c e s, ~ N a n j i n g ~ A g r i c u l t u r a l ~}$

17 University, Nanjing, China. 


\section{Abstract}

20 Graphene oxide (GO) is an oxidized form of graphene that is relatively cheap and easy to

21 produce. This has heralded its widespread use in a range of industries, with its likelihood of

22 release into the environment increasing accordingly. In pure culture, GO has been shown to

23 influence bacteria and fungi, but its effects on environmental microbial communities remain

24 poorly characterized, despite the important ecosystem services that these organisms underpin.

25 Here, we characterized the effects of GO and graphite, over time and at three concentrations (1

$26 \mathrm{ng}, 1 \mu \mathrm{g}$ and $1 \mathrm{mg} \mathrm{kg}$ dry soil ${ }^{-1}$ ), on soil bacterial and fungal diversity using $16 \mathrm{~S}$ rRNA and ITS2

27 gene amplicon sequencing. Graphite was included as a reference material as it is widely

28 distributed in the environment. Neither GO or graphite had significant effects on the alpha

29 diversity of microbial communities. The composition of bacterial and fungal communities,

30 however, was significantly influenced by both materials at all doses. Nonetheless, the effects of

31 GO and graphite were of similar magnitude, albeit with some differences in the taxa affected.

33 Keywords: phylogenetic marker gene sequencing, nanoparticles, microbial diversity,

34 nanotechnology 


\section{Introduction}

37 Graphene oxide (GO) is a nanomaterial with unique properties that has a wide-range of uses

38 (Novoselov et al., 2012; Yoo et al., 2014). The environmental release of GO is predicted to

39 increase along with the concomitant need to understand its potential ecological effects (Ahmed

40 and Rodrigues, 2013). Studies based on pure bacterial and fungal cultures indicate that GO can

41 elicit both positive and negative effects on cell integrity and function. For example, GO can kill

42 or suppress bacterial (Akhavan and Ghaderi, 2010; Liu et al., 2011) and fungal cultures (Chen et

43 al., 2014; Li et al., 2015), by inducing oxidative stress (Ahmed and Rodrigues, 2013; Liu et al.,

44 2011) or penetrating cell-walls and extracting phospholipids (Tu et al., 2013). On the other hand,

45 some studies have shown that GO can act as a respiratory electron acceptor (Salas et al., 2010;

46 Wang et al., 2011) and promote the growth of bacterial cultures (Ruiz et al., 2011). These studies

47 highlight that GO has the potential to affect soil microbial communities, which play fundamental

48 roles in the provision of ecosystem goods and services (Bardgett and van der Putten, 2014; Wagg

49 et al., 2014). Despite this, the impacts of GO on soil microbial diversity and function are poorly

50 understood.

52 To the best of our knowledge, there are only four previous studies that have directly investigated

53 the effects of GO on soil microbial communities (Chung et al., 2015; Du et al., 2015; Kim et al.,

54 2018; Xiong et al., 2018). The first, focused exclusively on soil microbial biomass and enzyme

55 activities (Chung et al., 2015). It revealed significant, but temporary, effects on the activities of

56 xylosidase, $\beta-1,4-\mathrm{N}$-acetyl glucosaminidase, and acid phosphatase, and no effects on microbial

57 biomass (Chung et al., 2015). The second study aimed to compare the diversity of bacterial 
communities in control and GO-amended soils using phylogenetic marker gene sequencing (Du

et al., 2015). The authors claimed that GO increased soil bacterial diversity and altered

their claims were unjustified as their study lacked replication within treatments $(n=1)$, and did not

account for differences in the numbers of sequences per sample when comparing the numbers of

taxa present. The third study investigated the effects of GO on microbial enzyme activities and

bacterial diversity in cadmium contaminated soil (Xiong et al., 2018). It revealed that the

activities of catalase and dehydrogenase increased, while that of urease decreased in response to

GO (Xiong et al., 2018). Furthermore, the composition of bacterial communities, but not their

67 diversity, appeared to be influenced by GO, although no statistical support for this was provided

68 (Xiong et al., 2018). Lastly, the fourth study focused on the effects of silver-GO nanocomposites

69 on soil bacterial communities, but included a GO treatment (Kim et al., 2018). Unfortunately, this

70 GO treatment was not compared to a water-only control; therefore, it was not possible to isolate

71 GO effects from those attributable to Ag (Kim et al., 2018).

73 Importantly, all previous studies applied GO at doses that are considerably higher than those

74 likely to be encountered in the environment. The main routes of entry for many nanomaterials

75 into the environment are thought to be wastewater treatment and landfill (Gottschalk et al., 2009;

76 Mueller and Nowack, 2008; Sun et al., 2014). While data are not available for GO, models

77 estimate that 3.7-7.1 ng and 0.8-1.6 $\mu \mathrm{g}$ of carbon nanotubes (CNTs) $\mathrm{kg} \mathrm{soil}^{-1}$ enter European

78 natural and sludge-amended soils every year (Sun et al., 2014). Model estimates for the rate of

79 release of other nanomaterials into soils (nanosilver, nano- $\mathrm{ZnO}$ and fullerenes) are similar (Sun et

80 al., 2014). Chung et al. (2015) applied 0.1-1.0 g GO kg soil ${ }^{-1}$, while Xiong et al. (2018) and Du et 
81 al. (2015) applied 1.0-2.0 $\mathrm{g}$ and $5 \mathrm{~g} \mathrm{GO} \mathrm{kg} \mathrm{soil}{ }^{-1}$, respectively. These doses are equivalent to

82 between 62.5 thousand and 1.25 billion times the estimated annual rate of accumulation for CNTs

83 (Sun et al., 2014). Hence, the effects of GO on soil microbial diversity are poorly understood,

84 particularly at concentrations approximating those deemed realistic for similar nanomaterials. In

85 addition, as previous studies have only considered bacterial communities, the effects of GO on

86 other important groups of soil organisms, such as fungi, are not known.

88 Finally, when characterizing the effects of nanomaterials on soil microbial diversity it is

89 important to include reference materials that provide a context with which to interpret changes

90 (Petersen, 2015). For example, while a significant shift in microbial diversity may be observed in

91 response to a nanomaterial, the magnitude of such a change may be small in comparison to those

92 associated with material that are already widely distributed in the environment. Clearly, therefore,

93 this information is essential for the development of appropriate policy frameworks for the safe

94 and sustainable use of nanomaterials. Despite this, such reference materials were not included in

95 any of the previous studies concerning the effects of GO on soil microbial communities (Chung

96 et al., 2015; Du et al., 2015; Kim et al., 2018; Xiong et al., 2018).

98 In this study, we investigated the effects of one-off applications of GO and graphite on soil

99 bacterial and fungal diversity using high-throughput phylogenetic marker gene sequencing.

100 Graphite was included as a reference compound as it chemically similar to GO, without being

101 classed as a nanomaterial, and is widely distributed in the environment. GO and graphite were

102 applied at three concentrations ( $1 \mathrm{ng}, 1 \mu \mathrm{g}$ and $\left.1 \mathrm{mg} \mathrm{GO} \mathrm{kg} \mathrm{soil}{ }^{-1}\right)$ representing environmentally

103 relevant low, high and extremely high rates of release based on those predicted for CNTs (Sun et 
104 al., 2014). All treatments were replicated three times and communities were characterized 7, 14

105 and 30 days after application.

106

\section{2. Materials and methods}

108

\subsection{Experimental design}

110 The soil used in this study is classified as a Kandosol according to the Australian Soil

111 Classification (Isbell, 2002), or an Ultisol according to the USDA Soil Taxonomy (Soil Survey

112 Staff, 2014) and has been described in our previous work (see Table S1 from Wang et al. (2016)).

113 Briefly, the soil was collected at a depth of 0-20 $\mathrm{cm}$ from a pineapple (Ananas comosus) farm in

114 Queensland, Australia. The soil had a sandy loam texture, $\mathrm{pH}$ of 5.4 (1:5 soil/water), conductivity

115 of $0.1 \mathrm{dS} / \mathrm{m}$ (1:5 soil/water), and total organic Carbon content of $1.1 \%$. Approximately $11 \mathrm{~kg}$ of

116 fresh soil was passed through a $2 \mathrm{~mm}$ sieve and then split into seven c. $1.6 \mathrm{~kg}$ sub-samples to

117 which the treatments were applied. Graphene oxide (Aldrich Chemistry, CAT: 763705, Lot\#

118 MKBQ8029V) and graphite (Sigma Aldrich, CAT:282863, size <20 $\mu \mathrm{m}$ ) were applied to soils at

119 rates of $1 \mathrm{ng}, 1 \mu \mathrm{g}$ and $1 \mathrm{mg} \mathrm{kg}$ dry soil ${ }^{-1}$ using a fine mist sprayer and thoroughly homogenized

120 by mechanical mixing. In brief, either the GO or graphite was dispersed into deionized water at a

121 volume that resulted in the soil being adjusted to $50 \%$ water holding capacity (WHC). The

122 solution was then sprayed on the soil prior to mechanical mixing. These three rates were selected

123 to represent environmentally relevant, high and extremely high concentrations based on release

124 rates predicted for CNTs (Sun et al., 2014). Soils in the control treatments were sprayed with an

125 equal quantity of deionized water only and then mixed in the same manner as for the GO and

126 graphite treatments. Three replicate $500 \mathrm{~g}$ samples of each treatment were placed into $1 \mathrm{~L}$ plastic 
containers with lids that facilitated gas exchange. This yielded 21 containers that were incubated

128 for 30 days in the dark at $25^{\circ} \mathrm{C}$, with the humidity maintained at $80 \%$ in order to keep the soils at

129 the same WHC throughout the experimental period.

130

\subsection{Soil sampling and DNA extraction}

132 Soil cores, of approximately $25 \mathrm{~g}$, were collected from each experimental unit after 7, 14 and 30

133 days using sterile $50 \mathrm{ml}$ plastic tubes, and immediately transferred to $-80^{\circ} \mathrm{C}$ storage. DNA was

134 extracted from $250 \mathrm{mg}$ of thawed soil using the Power Soil DNA Isolation kit (MO BIO

135 Laboratories, Carlsbad, CA) according to the manufacturers' instructions. To avoid systematic

136 biases we randomized the order of samples for all processing steps.

\subsection{PCR amplification and sequencing of phylogenetic marker genes}

139 Universal bacterial 16S rRNA genes were amplified by polymerase chain reaction (PCR) using

140 the primers 926F (5'-AAA CTY AAA KGA ATT GRC GG-3') (Engelbrektson et al., 2010) and

141 1392wR (5'-ACG GGC GGT GWG TRC-3') (Engelbrektson et al., 2010). Fungal ITS2 regions

142 were PCR amplified using the primers gITS7F (5'- GTG ART CAT CGA RTC TTT G -3')

143 (Ihrmark et al., 2012) and ITS4R (5'- TCC TCC GCT TAT TGA TAT GC -3') (White et al.,

144 1990). For both groups, the forward and reverse primers were modified on the 5 ' end to contain

145 the Illumina overhang adapter for compatibility with the i5 and i7 Nextera XT indices,

146 respectively. PCRs were performed with $1.5 \mu$ l template DNA, in 1X PCR Buffer minus $\mathrm{Mg}^{2+}$

147 (Invitrogen), $100 \mu \mathrm{M}$ of each of the dNTPs (Invitrogen), $300 \mu \mathrm{M}$ of $\mathrm{MgCl}_{2}$ (Invitrogen), $0.625 \mathrm{U}$

148 Taq DNA Polymerase (Invitrogen), and $250 \mu \mathrm{M}$ of each primer, made up to a total volume of 25

$149 \mu \mathrm{l}$ with molecular biology grade water. Thermocycling conditions were as follows: $94^{\circ} \mathrm{C}$ for 3 
150 minutes; then 35 cycles of $94^{\circ} \mathrm{C}$ for 45 seconds, $55^{\circ} \mathrm{C}$ for 30 seconds, $72^{\circ} \mathrm{C}$ for 1 minute 30

151 seconds; followed by $72^{\circ} \mathrm{C}$ for 10 minutes. Amplifications were performed using a Veriti ${ }^{\circledR} 96$ -

152 well Thermocycler (Applied Biosystems). PCR success was determined by gel electrophoresis,

153 which also facilitated visual confirmation of amplicon size and quality.

155 Amplicons were purified using AMPure magnetic beads (Agencourt) and subjected to dual

156 indexing using the Nextera XT Index Kit (Illumina) as per the manufacturer's instructions.

157 Indexed amplicons were purified using AMPure XP beads and then quantified using a PicoGreen

158 dsDNA Quantification Kit (Invitrogen). Equal concentrations of each sample were pooled and

159 sequenced on an Illumina MiSeq at The University of Queensland's Institute for Molecular

160 Biosciences (UQ, IMB) using 30\% PhiX Control v3 (Illumina) and a MiSeq Reagent Kit v3 (600

161 cycle; Illumina) according the manufacturer's instructions.

\subsection{Processing of sequence data}

164 Data were analyzed using a modified UPARSE pipeline (Edgar, 2013). For both datasets, 165 analyses were performed using the forward reads only. For 16S rDNA sequences, USEARCH

166 (v10.0.240) (Edgar, 2010) was used to perform the following steps: 1) primers were removed and

167 the residual sequences were trimmed to 250 bp using fastx_truncate; 2) high-quality sequences

168 were identified using fastq_filter by discarding reads with greater than one expected error (-

169 fastq_maxee=1); 3) duplicate sequences were removed using fastx_uniques; 4) sequences were

170 clustered at $97 \%$ similarity into operational taxonomic units (OTU) and potential chimeras were

171 identified and removed using cluster_otus; and 5) an OTU table was generated using otutab with

172 default parameters from the pre-trimmed reads and the OTU representative sequences. For the 
173 ITS data, ITSx v1.0.11 (Bengtsson-Palme et al., 2013) was used to identify and extract fungal

174 ITS2 sequences. Chimeric ITS2 sequences were identified and removed using the uchime2_ref

175 command of USEARCH and the UNITE database (v7.2 - 2017.10.10) (Nilsson et al., 2019).

176 ITS2 sequences were then clustered at 97\% similarity into operational taxonomic units (OTU)

177 and an OTU table was generated using the otutab command of USEARCH with default

178 parameters. SILVA SSU (v128) (Quast et al., 2013) and UNITE (v7.2-2017.10.10) (Nilsson et

179 al., 2019) taxonomy was assigned to the 16 S and ITS sequences, respectively, using BLASTN

180 (v2.3.0+) (Zhang et al., 2000) within the feature classifier of QIIME2 (v2017.9) (Boylen et al.,

181 2018). The $16 \mathrm{~S}$ OTU table was then filtered to remove OTUs classified as chloroplasts,

182 mitochondria, archaea or eukaryotes using the BIOM (McDonald et al., 2012) tool suite. For 16S,

183 de-novo multiple sequence alignments of the representative OTU sequences were generated using

184 MAFFT (v7.221) (Katoh and Standley, 2013) and masked with the alignment mask command of

185 QIIME2. The masked alignment was used to generate a midpoint-rooted phylogenetic tree using

186 FastTree (v2.1.9) (Price et al., 2010) in QIIME2. OTU tables were rarefied to 4850 and 1150

187 sequences per sample for 16 S and ITS, respectively. The mean numbers of observed (Sobs) and

188 predicted (Chao1) OTUs were calculated using QIIME2 for both bacteria and fungi. For bacteria,

189 we also calculated Faith's phylogenetic diversity index (Faith's PD) (Faith, 1992) using QIIME2.

\subsection{Statistical analyses}

192 For statistical analyses, we defined Treatment as the combination of applied substance (none for

193 the control, GO or graphite) and applied dose $\left(1 \mathrm{ng}, 1 \mu \mathrm{g}\right.$ or $1 \mathrm{mg} \mathrm{kg}$ dry soil $\left.{ }^{-1}\right)$. Hence,

194 Treatment was defined as a categorical variable with seven classes. In order to determine whether

195 the GO and graphite treatments significantly affected the alpha diversity metrics, we used a linear 
196 mixed-effects model approach (Pinheiro and Bates, 2004). Treatment (as defined above) and

197 Day, as well as their interaction, were treated as fixed effects, and soil containers (samples) were

198 treated as a random effect to account for the repeated measures. F-tests were applied to assess

199 significance $(P<0.05)$, and were implemented in $\mathrm{R}$ using the lme4 (Bates et al., 2015) and

200 lmerTest (Kuznetsova, 2017) packages.

201

202 Differences in the relative abundances of taxa between samples (beta diversity), and the inferred

203 functional profiles were assessed using multivariate generalized linear models using a negative

204 binomial distribution (Warton, 2011). The significance of differences in community composition

205 was determined by comparing the sum-of-likelihood test statistics for the alternative statistical

206 models via a resampling method (Wang et al., 2012) that accounted for the correlation between

207 species and the correlation within the repeated measures taken from the same sample container.

208 These comparisons were implemented in R using the mvabund package (Wang et al., 2012). Taxa

209 whose maximum relative abundance was less than $0.1 \%$ for bacteria or $0.4 \%$ for fungi were

210 disregarded before statistical analysis. Where an interactive effect of Treatment and Day was

211 significant, post-hoc analyses were undertaken to investigate which Treatments differed on what

212 Days. Where no interactive effect was found, but a main effect of Treatment was, post-hoc

213 analyses focused on which Treatments differed from one another. The Benjamini-Hochberg

214 correction was applied to all post-hoc tests.

2162.6 Data availability

217 All sequences have been deposited to the Sequence Read Archive (SRA) under BioProject

218 accession number GenBank: PRJNA515098. 


\section{3. Results}

\subsection{Alpha diversity of soil microbial communities}

223 The numbers of observed (Sobs) and predicted (Chao1) bacterial and fungal taxa, as well as the

224 phylogenetic diversity of bacterial communities (Faith's PD), were not significantly influenced

225 by any of the GO or graphite treatments relative to the controls throughout the experiment (Fig.

226 1, S1).

\subsection{Soil bacterial community composition}

229 Relative to the controls, the composition of soil bacterial communities was significantly

230 influenced by the addition of GO and graphite, and these effects differed over time $(P<0.001)$.

231 Effects were observed for both materials at all doses except for the low GO dose on day 14

232 (Tables 1 and S1). Albeit significant, there was not a consistent direction of change in bacterial

233 community composition with increasing GO or graphite dose (Fig. 2). Bacterial community

234 composition differed significantly between all GO and graphite treatments (Table S2).

236 Bacterial communities were dominated by representatives of the Acidobacteria, Actinobacteria,

237 Armatimonadetes, Bacteriodetes, Candidatus Berkelbacteria, Chlamydiae, Chlorobi, Chloroflexi,

238 Fibrobacteres, Firmicutes, Gemmatimonadetes, Microgenomates, Planctomycetes and

239 Proteobacteria, Saccharibacteria, and Verrucomicrobia (Fig. S2). 
241 The 100 OTUs that were most strongly associated with differences in community composition

242 between treatments were obtained from the multivariate generalized linear models (GLMs) and

243 assessed independently using univariate GLM models. Of these 49 were found to differ

244 significantly from the control in at least one treatment combination after Benjamini-Hochberg

245 correction for multiple comparisons (Fig. 3). Four OTUs responded exclusively to GO: a member

246 of the Tepidisphaeraceae (OTU81, Planctomycetes) and an Oligoflexales (OTU363,

247 Deltaproteobacteria) that increased in relative abundance in the presence of GO; a

248 Ktedonobacteria (OTU1084, Chloroflexi) population which declined; and a representative of the

249 Acidobacteriaceae (OTU2196, Acidobacteria), which increased at some doses but declined at

250 others (Fig. 3). An additional 30 OTUs, representing a broad-range of phyla, responded to both

251 GO and graphite (Fig. 3). The remaining 15 OTUs, again of broad phylogenetic coverage,

252 responded exclusively to graphite (Fig. 3).

\subsection{Soil fungal community composition}

255 Relative to the controls, the composition of soil fungal communities was significantly influenced

256 by the addition of GO and graphite at all doses $(P=0.001)$, and these treatment effects did not

257 differ significantly over time (Tables 2 and S3). As observed for bacteria, there was not a

258 consistent direction of change in the composition of fungal communities with increasing GO or

259 graphite dose (Fig. 4). Fungal community composition differed significantly between all GO and

260 graphite treatments (Table S3).

261

262 Soil fungal communities were dominated by representatives of the Ascomycota, Basidiomycota,

263 Mortierellomycota, Mucoromycota and Chytridiomycota (Fig. S3). As for the bacteria, the fungal 
264 OTUs that were most strongly associated with differences in community composition between

265 treatments were obtained from the multivariate GLMs and assessed independently using

266 univariate GLM models. Of these, 16 OTUs were found to differ significantly from the control in

267 at least one treatment combination after Benjamini-Hochberg correction for multiple comparisons

268 (Fig. 5).

270 No fungal OTUs responded exclusively to GO; however, 10 responded to both GO and graphite

271 (Fig. 5). In all cases the direction of response for these OTUs was the same for GO as it was for

272 graphite. Four of these OTUs were negatively associated with GO and graphite viz. a

273 Goidanichiella sphaerospora (OTU 10), a Pleosporales (OTU 87), a Kendrickiella phycomyces

274 (OTU 16) and a Hendersonia (OTU 41) population (Fig. 5). In contrast, the relative abundances

275 of six OTUs were positively associated with GO and graphite: an Arthrobotrys musiformis (OTU

276 17), a Trichoderma virens (OTU 136), a Saitozyma podzolica (OTU 62), a Ceratocystidaceae

277 (OTU 3), a Clonostachys candelabrum (OTU 25), and a Fusarium (OTU 422) population (Fig.

278 5). The remaining six OTUs responded exclusively to graphite with two decreasing (OTUs 85

279 and 39) and four increasing (OTUs 53, 56, 75, and 58) in relative abundance, respectively (Fig.

$2805)$.

281

\section{4. Discussion}

284 Our study demonstrates that GO can significantly alter the composition, but not alpha diversity,

285 of soil bacterial and fungal communities at loading rates equal to, and beyond, those estimated for

286 the annual accumulation of other nanomaterials (e.g. carbon nanotubes, nanosilver, nano- $\mathrm{TiO}_{2}$ 
and nano-ZnO) in soils (Sun et al., 2014). Nonetheless, our reference material, graphite, also led

to significant shifts in community composition, and these were of similar magnitude to those

observed for GO, albeit with some differences in the taxa affected.

Of the four previous studies that investigated the effects of GO on soil microbial communities

292 (Chung et al., 2015; Du et al., 2015; Kim et al., 2018; Xiong et al., 2018), three considered

293 bacterial diversity (Du et al., 2015; Kim et al., 2018; Xiong et al., 2018) and two measured

294 microbial enzyme activities (Chung et al., 2015; Xiong et al., 2018). In all cases, the results for

295 bacterial diversity were inconclusive due to a lack of replication (Du et al., 2015), no treatment

296 controls (Kim et al., 2018) or statistical analyses (Du et al., 2015; Xiong et al., 2018). The

297 activities of several enzymes, however, were significantly, albeit only temporally, influenced by

298 GO addition (Chung et al., 2015; Xiong et al., 2018), suggesting that GO has at least some

299 potential to influence soil ecosystem functioning. Nonetheless, all previous studies have focused

300 on the effects of extremely high GO doses - equivalent to between 62.5 thousand and 1.25 billion

301 times the estimated annual rate of accumulation for CNTs (Sun et al., 2014). It could be argued,

302 therefore, that smaller doses would have lesser effects on microbial communities.

304 Interestingly, our study highlights that the magnitude of community compositional changes

305 observed did not increase with the amount of material applied, or decrease over time. This

306 indicates that even low GO doses (i.e. parts per trillion to parts per billion) can induce community

307 compositional changes that persist beyond 30 days. We speculate that this lack of 'linear' dose-

308 response to GO is related to an agglomeration of GO sheets (Dreyer et al., 2010), covering of

309 sheets with other materials (Hui et al., 2014), or interactions with components of the soil matrix 
310 (Chen et al., 2018), such as clays which possess a large surface area, capable of binding and

311 immobilizing GO sheets (T. Lu et al., 2017). These interactions could strongly influence the

312 number of GO sheets that interact with soil microbes and significantly alter the properties that

313 they exhibit as pristine nanoparticles. For example, culture-based studies have demonstrated that

314 the edges of GO sheets can cut and destructively extract phospholipids (X. Lu et al., 2017; Tu et

315 al., 2013) from microbial cell membranes. This destructive property of GO is likely to be

316 undermined by interactions that would reduce the number of 'sharp' edges (Akhavan and

317 Ghaderi, 2010; Liu et al., 2011).

319 While there were significant differences in community composition between GO and graphite 320 amended soils, the majority of OTUs that discriminated between treatments, responded in a

321 similar manner to both materials. For example, $61 \%$ and $63 \%$ of discriminating bacterial and

322 fungal taxa, respectively, responded to both GO and graphite. In contrast, just $8 \%$ of

323 discriminating bacterial taxa, and no fungal taxa, responded exclusively to GO; while $31 \%$ and

$32438 \%$ of discriminating bacterial and fungal taxa, respectively, responded exclusively to graphite.

325 In general, there were roughly equal numbers of positive and negative responses for taxa

326 responding to GO and/or graphite. This observation corroborates pure culture studies, which

327 report both positive and negative effects of GO on bacterial and fungal isolates (Ahmed and

328 Rodrigues, 2013; Akhavan and Ghaderi, 2010; Chen et al., 2014; Li et al., 2015; Liu et al., 2011;

329 Ruiz et al., 2011; Salas et al., 2010; Tu et al., 2013; Wang et al., 2011). One of the ways in which

330 GO may benefit microbes is by acting as a terminal electron acceptor (Salas et al., 2010; Wang et

331 al., 2011); however, we did not find evidence that respiratory pathways were affected by GO and

332 did not detect any taxa that are well-known to perform extracellular electron transfer. In 
333 summary, fewer populations responded exclusively to GO than to graphite; most responded to

334 both materials; and the numbers of positively and negatively affected taxa were about equal.

336 Despite lacking replication and/or statistical analyses, previous studies that investigated the

337 effects of GO on soil microbial diversity have drawn attention to apparent differences in the

338 relative abundances of ecologically significant taxa, such as those involved in nitrogen cycling.

339 Xiong et al. (2018), for example, reported an increase in the relative abundance of Rhizobiales

340 populations and decreases in the relative abundances of Rhodospirrillaeceae and Nitrospirae. In

341 our study, just one Rhizobiales (OTU 195) population was affected by GO addition, and this

342 effect manifested as a decrease in its relative abundance at 7 days, followed by an increase after

34314 days. Among the Rhodospirrilaeceae, we observed an increase in the relative abundance of

344 OTU 1929, and a decrease in the relative abundance of OTU 819 in response to GO addition

345 (Fig. 3). We did not detect any significant changes in the relative abundances of members of the

346 Nitrospirae in response to GO or graphite. To the best of our knowledge, the effects of GO on

347 soil fungal communities have not been previously examined. In pure culture, GO has been shown

348 to suppress the growth of Fusarium oxysporum, a species known to contain multiple plant

349 pathogens (Chen et al., 2014). Despite being present within our inventories, we did not detect

350 significant effects of GO on this species; however, the relative abundance of an unclassified

351 relative within the same genus (OTU 422) was observed to increase in response to both GO and

352 graphite addition (Fig. 5).

353

354 5. Conclusion 
356 As the use of GO increases, its release into the environment will rise. Our study demonstrates that

357 that GO and graphite can influence soil bacterial and fungal community composition, but that

358 their effects are of similar magnitude, albeit with some differences in the taxa affected. In light of

359 this finding, it is important that future studies examine whether GO-induced changes in microbial

360 diversity are likely to undermine the provision of soil ecosystem goods and services.

361

362 Acknowledgements

364 The authors gratefully acknowledge financial support from The University of Queensland for an

365 Early Career Researcher Award to PGD. CF gratefully acknowledges funding from the

366 Australian Government's Department of Education and Training in the form of an Australian

367 Government Research Training Program Scholarship administered by The University of

368 Queensland. PMK is the recipient of an Australian Research Council (ARC) Future Fellowship

369 (ARC FT120100277).

370

371 Declaration of interest

372

373 The authors report that they have no conflicts of interest.

\section{References}

376

377 Ahmed, F., Rodrigues, D.F., 2013. Investigation of acute effects of graphene oxide on

378 wastewater microbial community: A case study. J. Hazard. Mater. 256-257, 33-39. 
Akhavan, O., Ghaderi, E., 2010. Toxicity of graphene and graphene oxide nanowalls against bacteria. ACS Nano 4, 5731-5736. https://doi.org/10.1021/nn101390x

Bardgett, R.D., van der Putten, W.H., 2014. Belowground biodiversity and ecosystem functioning. Nature 515, 505-511. https://doi.org/10.1038/nature13855 using lme4. J. Stat. Softw. 67, 1-48. https://doi.org/10.18637/jss.v067.i01 Sánchez-García, M., Ebersberger, I., de Sousa, F., Amend, A., Jumpponen, A., Unterseher, ITS2 from ribosomal ITS sequences of fungi and other eukaryotes for analysis of environmental sequencing data. Methods Ecol. Evol. 4, 914-919.

Boylen, E., Rideout, J.R., Dillon, M.R., Bokulich, N.A., Abnet, C., Al-Ghalith, G.A., Alexander, https://doi.org/10.1111/2041-210X.12073 

interactive, scalable, and extensible microbiome data science. PeerJ Prepr. 6e27295v2 https//doi.org/10.7287/peerj.preprints.27295v2.

Chen, J., Peng, H., Wang, X., Shao, F., Yuan, Z., Han, H., 2014. Graphene oxide exhibits broadspectrum antimicrobial activity against bacterial phytopathogens and fungal conidia by intertwining and membrane perturbation. Nanoscale 6, 1879-1889.

Chen, L., Yang, S., Liu, Y., Mo, M., Guan, X., Huang, L., Sun, C., Yang, S., Chang, X., 2018. https://doi.org/10.1039/c3nr04941h

419 Chung, H., Kim, M.J., Ko, K., Kim, J.H., Kwon, H., Hong, I., Park, N., Lee, S., Kim, W., 2015. 420 Effects of graphene oxides on soil enzyme activity and microbial biomass. Sci. Total Environ. 514, 307-313. https://doi.org/10.1016/j.scitotenv.2015.01.077

422 Dreyer, D.R., Park, S., Bielawski, C.W., Ruoff, R.S., 2010. The chemistry of graphene oxide. 
424 Du, J., Hu, X., Zhou, Q., 2015. Graphene oxide regulates the bacterial community and exhibits

Edgar, R.C., 2013. UPARSE: Highly accurate OTU sequences from microbial amplicon reads. 26, 2460-2461. https://doi.org/10.1093/bioinformatics/btq461

Engelbrektson, A., Kunin, V., Wrighton, K.C., Zvenigorodsky, N., Chen, F., Ochman, H., regions. Environ. Sci. Technol. 43, 9216-9222. https://doi.org/10.1021/es9015553 
445 Ihrmark, K., Bödeker, I.T.M., Cruz-Martinez, K., Friberg, H., Kubartova, A., Schenck, J., Strid,

446 Y., Stenlid, J., Brandström-Durling, M., Clemmensen, K.E., Lindahl, B.D., 2012. New

447 primers to amplify the fungal ITS2 region - evaluation by 454-sequencing of artificial and

448 natural communities. FEMS Microbiol. Ecol. 82, 666-677. https://doi.org/10.1111/j.1574-

$449 \quad$ 6941.2012.01437.x

450 Isbell, R.F., 2002. The Australian soil classification (Revised ed.). CSIRO Publishing,

$451 \quad$ Collingwood, Victoria.

452 Katoh, K., Standley, D.M., 2013. MAFFT multiple sequence alignment software version 7:

453 Improvements in performance and usability. Mol. Biol. Evol. 30, 772-780.

454 https://doi.org/10.1093/molbev/mst010

455 Kim, M., Ko, D., Ko, K., Kim, D., Lee, J., Woo, S.M., Kim, W., Chung, H., 2018. Effects of

456 silver-graphene oxide nanocomposites on soil microbial communities. J. Hazard. Mater.

457 346, 93-102. https://doi.org/10.1016/j.jhazmat.2017.11.032

458 Kuznetsova, A., 2017. lmerTest: Tests in linear mixed effects models. R package version 3.0-1. J.

459 Stat. Softw. 82, 1-26.

460 Li, X., Li, F., Gao, Z., Fang, L., 2015. Toxicology of graphene oxide nanosheets against

461 Paecilomyces catenlannulatus. Bull. Environ. Contam. Toxicol. 95, 25-30.

462 https://doi.org/10.1007/s00128-015-1499-3

463 Liu, S., Zeng, T.H., Hofmann, M., Burcombe, E., Wei, J., Jiang, R., Kong, J., Chen, Y., 2011.

464 Antibacterial activity of graphite, graphite oxide, graphene oxide, and reduced graphene 
oxide: Membrane and oxidative stress. ACS Nano 5, 6971-6980. https://doi.org/10.1021/nn202451x

Lu, T., Xia, T., Qi, Y., Zhang, C., Chen, W., 2017. Effects of clay minerals on transport of graphene oxide in saturated porous media. Environ. Toxicol. Chem. 36, 655-660. https://doi.org/10.1002/etc.3605 Enhanced antibacterial activity through the controlled alignment of graphene oxide nanosheets. Proc. Natl. Acad. Sci. U S A E9793-E9801.

McDonald, D., Clemente, J.C., Kuczynski, J., Rideout, J.R., Stombaugh, J., Wendel, D., Wilke, Observation Matrix (BIOM) format or: How I learned to stop worrying and love the omeenvironment. Environ. Sci. Technol. 42, 4447-4453. https://doi.org/10.1021/es7029637 ome. Gigascience 1, 1-6. https://doi.org/10.1186/2047-217X-1-7 Kennedy, P., Picard, K., Glöckner, F.O., Tedersoo, L., Saar, I., Koljalg, U., Abarenkov, K., 2019. The UNITE database for molecular identification of fungi: handling dark taxa and parallel taxonomic classifications. Nucleic Acids Res. 47, D259-D264. https://doi.org/10.1016/0038-1098(79)91043-3

Novoselov, K.S., Fal'ko, V.I., Colombo, L., Gellert, P.R., Schwab, M.G., Kim, K., 2012. A roadmap for graphene. Nature 490, 192-200. https://doi.org/10.1038/nature11458 
Petersen, E.J., 2015. Control experiments to avoid artifacts and misinterpretations in nanoecotoxicology testing. NIST Spec. Publ. 1200, 1-7. https://doi.org/http://dx.doi.org/10.6028/NIST.SP.1200-11

490 Pinheiro, J.C., Bates, D.M., 2004. Mixed-effects models in S and S-Plus. Springer, New York, 491 USA.

Price, M.N., Dehal, P.S., Arkin, A.P., 2010. FastTree 2--approximately maximum-likelihood trees for large alignments. PLoS One 5, e9490. https://doi.org/10.1371/ journal.pone.0009490

Quast, C., Pruesse, E., Yilmaz, P., Gerken, J., Schweer, T., Yarza, P., Peplies, J., Glöckner, F.O.F.O., 2013. The SILVA ribosomal RNA gene database project: improved data processing and web-based tools. Nucleic Acids Res. 41, D590-D596. https://doi.org/10.1093/nar/gks1219

Ruiz, O.N., Fernando, K.A.S., Wang, B., Brown, N.A., Luo, P.G., McNamara, N.D., Vangsness,

$500 \quad$ M., Sun, Y.-P., Bunker, C.E., 2011. Graphene oxide: A nonspecific enhancer of cellular growth. ACS Nano 5, 8100-8107. https://doi.org/10.1021/nn202699t

502 Salas, E.C., Sun, Z., Luttge, A., Tour, J.M., Lüttge, A., Tour, J.M., Lüttge, A., Tour, J.M., 2010.

503 Reduction of Graphene Oxide via Bacterial Respiration. ACS Nano 4, 4852-4856.

$504 \quad$ https://doi.org/10.1021/nn101081t

505 Soil Survey Staff, 2014. Keys to soil taxonomy, 12th ed. USDA - Natural Resources

506 Conservation Service, Washington, DC.

507 Sun, T.Y., Gottschalk, F., Hungerbühler, K., Nowack, B., 2014. Comprehensive probabilistic 
modelling of environmental emissions of engineered nanomaterials. Environ. Pollut. 185, 69-76. https://doi.org/10.1016/j.envpol.2013.10.004

Tu, Y., Lv, M., Xiu, P., Huynh, T., Zhang, M., Castelli, M., Liu, Z., Huang, Q., Fan, C., Fang, H., Zhou, R., 2013. Destructive extraction of phospholipids from Escherichia coli membranes by graphene nanosheets. Nat. Nanotechnol. 8, 594-601.

Wagg, C., Bender, S.F., Widmer, F., van der Heijden, M.G.A., 2014. Soil biodiversity and soil community composition determine ecosystem multifunctionality. Proc. Natl. Acad. Sci. U S A 111, 5266-5270. https://doi.org/10.1073/pnas.1320054111

Wang, Y., Naumann, U., Wright, S.T., Warton, D.I., 2012. Mvabund - an R package for modelbased analysis of multivariate abundance data. Methods Ecol. Evol. 3, 471-474. https://doi.org/10.1111/j.2041-210X.2012.00190.x generalized estimating equations. Biometrics 67, 116-123. https://doi.org/10.1111/j. 
529 White, T.J., Bruns, T., Lee, S., Taylor, J.W., 1990. Amplification and direct sequencing of fungal

530 ribosomal RNA genes for phylogenetics., in: Innis, M.A., Gelfand, D.H., Sninsky, J.J.,

531 White, T.J. (Eds.), PCR Protocols: A Guide to Methods and Applications. Academic Press,

$532 \quad$ New York, pp. 315-322.

533 Xiong, T., Yuan, X., Wang, H., Leng, L., Li, H., Wu, Z., Jiang, L., Xu, R., Zeng, G., 2018.

$534 \quad$ Implication of graphene oxide in Cd-contaminated soil: A case study of bacterial

535 communities. J. Environ. Manage. 205, 99-106.

536 https://doi.org/10.1016/j.jenvman.2017.09.067

537 Yoo, B.M., Shin, H.J., Yoon, H.W., Park, H.B., 2014. Graphene and graphene oxide and their

538 uses in barrier polymers. J. Appl. Polym. Sci. 131, 1-23. https://doi.org/10.1002/app.39628

539 Zhang, Z., Schwartz, S., Wagner, L., Miller, W., 2000. A greedy algorithm for aligning DNA

540 sequences. J. Comput. Biol. 7, 203-214. https://doi.org/10.1089/10665270050081478 
545 Table 1 Summary of multivariate GLM post-hoc results computed using mvabund highlighting

546 differences in bacterial community composition between treatments relative to the controls within

547 each time point.

549 Table 2 Summary of multivariate GLM post-hoc results computed using mvabund highlighting

550 differences in fungal community composition between treatments relative to the controls.

551

552 Figure Legends

553

554 Fig. 1 The numbers of predicted bacterial and fungal OTUs (Chao1) after 7, 14 and 30 days by

555 treatment. The error bars represent standard deviations. None of the treatments differed

556 significantly from the controls. GO and graphite doses correspond to $1 \mathrm{ng}, 1 \mu \mathrm{g}$ and $1 \mathrm{mg} \mathrm{kg}^{-1}$

557 soil.

558

559 Fig. 2 Principal coordinate analysis (PCoA) ordination illustrating differences in the composition

560 of bacterial communities in control, and graphite and GO amended soils over time. The ellipses

561 represent standard deviations. The three panels, representing different time points, derive from a

562 single ordination that included all samples. Each time point is shown separately to avoid over-

563 cluttering. The arrows for each treatment move from low, through medium, to high dose. They

564 highlight that there was not a consistent direction of change in community composition with 
565 increasing GO or graphite dose. GO and graphite doses correspond to $1 \mathrm{ng}, 1 \mu \mathrm{g}$ and $1 \mathrm{mg} \mathrm{kg}^{-1}$

566 soil.

568 Fig. 3 Heatmap of the relative abundances of 49 bacterial OTUs that differed significantly from

569 the control in at least one treatment combination. The asterisks highlight which treatments differ

570 significantly from the controls on each day $\left(P<0.05^{*}, P<0.01^{* *}, P<0.001^{* * *}\right)$. Each column

571 of the heatmap represents the mean relative abundance of each treatment $(n=3)$. The bubble-plot

572 on the left summarizes the number (circle size) of GO or graphite doses that an OTU responded

573 to relative to the controls, and of these how many manifested as increases or decreases in relative

574 abundance (circle color). The numbers below the bubble plot and heatmap show the total

575 numbers of significant responses to a particular treatment relative to the control within the same

576 day. The OTU IDs are consistent throughout the manuscript. The phylum of each OTU is

577 indicated by the colors on the left of the heatmap and the affiliations associated with each color

578 are shown at the bottom. GO and graphite doses correspond to $1 \mathrm{ng}, 1 \mu \mathrm{g}$ and $1 \mathrm{mg} \mathrm{kg}^{-1}$ soil.

580 Fig. 4 Principal coordinate analysis (PCoA) ordination illustrating differences in the composition

581 of fungal communities in control, and graphite and GO amended soils over time. The ellipses

582 represent standard deviations. The three panels, representing different time points, derive from a

583 single ordination that included all samples. Each time point is shown separately to avoid over-

584 cluttering. On day 30, one of the control samples $(\mathrm{PCO} 1=1.2, \mathrm{PCO} 2=-0.07)$ is beyond the

585 plotted area. The arrows for each treatment move from low, through medium, to high dose. They

586 highlight that there was not a consistent direction of change in community composition with 
587 increasing GO or graphite dose. GO and graphite doses correspond to $1 \mathrm{ng}, 1 \mu \mathrm{g}$ and $1 \mathrm{mg} \mathrm{kg}^{-1}$

588 soil.

590 Fig. 5 Heatmap of the relative abundances of 16 fungal OTUs that differed significantly from the

591 control in at least one treatment combination. The asterisks highlight which treatments differ

592 significantly from the controls on each day $\left(P<0.05^{*}, P<0.01^{* *}, P<0.001^{* * *}\right)$. Each column

593 of the heatmap represents the mean relative abundance of each treatment $(n=9)$. The bubble-plot

594 on the left summarizes the number (circle size) of GO or graphite doses that an OTU responded

595 to relative to the controls, and of these how many manifested as increases or decreases in relative

596 abundance (circle color). The numbers below the bubble plot and heatmap show the total

597 numbers of significant responses to a particular treatment relative to the control within the same

598 day. The OTU IDs are consistent throughout the manuscript. The phylum and class of each OTU

599 is indicated by the colors on the left of the heatmap and the affiliations associated with each color

600 are shown at the bottom. GO and graphite doses correspond to $1 \mathrm{ng}, 1 \mu \mathrm{g}$ and $1 \mathrm{mg} \mathrm{kg}^{-1} \mathrm{soil}_{\text {. }}$ 


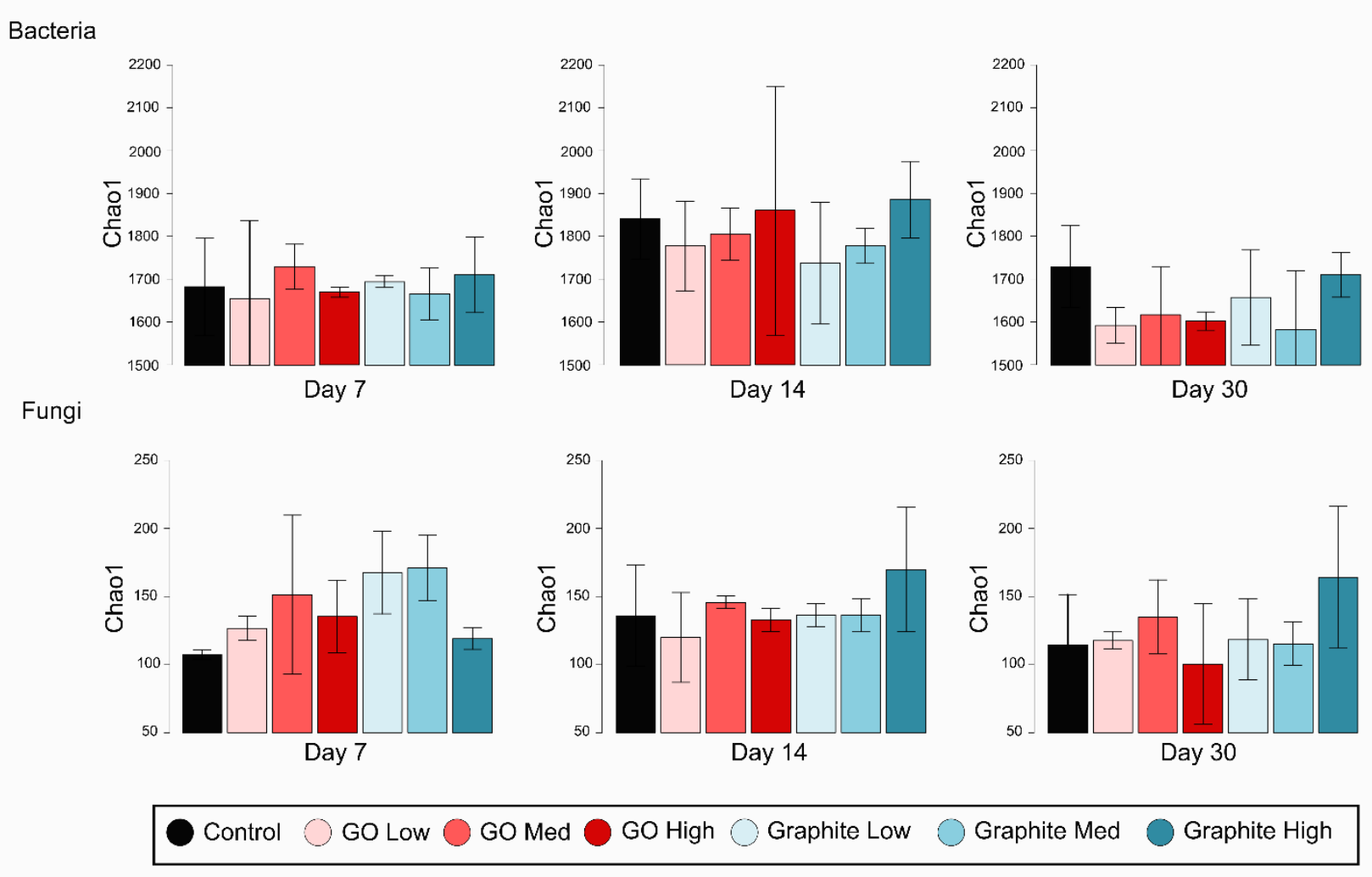

Fig. 1 The numbers of predicted bacterial and fungal OTUs (Chao1) after 7, 14 and 30 days by treatment. The error bars represent standard deviations. None of the treatments differed significantly from the controls. GO and graphite doses correspond to $1 \mathrm{ng}, 1 \mu \mathrm{g}$ and $1 \mathrm{mg} \mathrm{kg}^{-1}$ soil. 


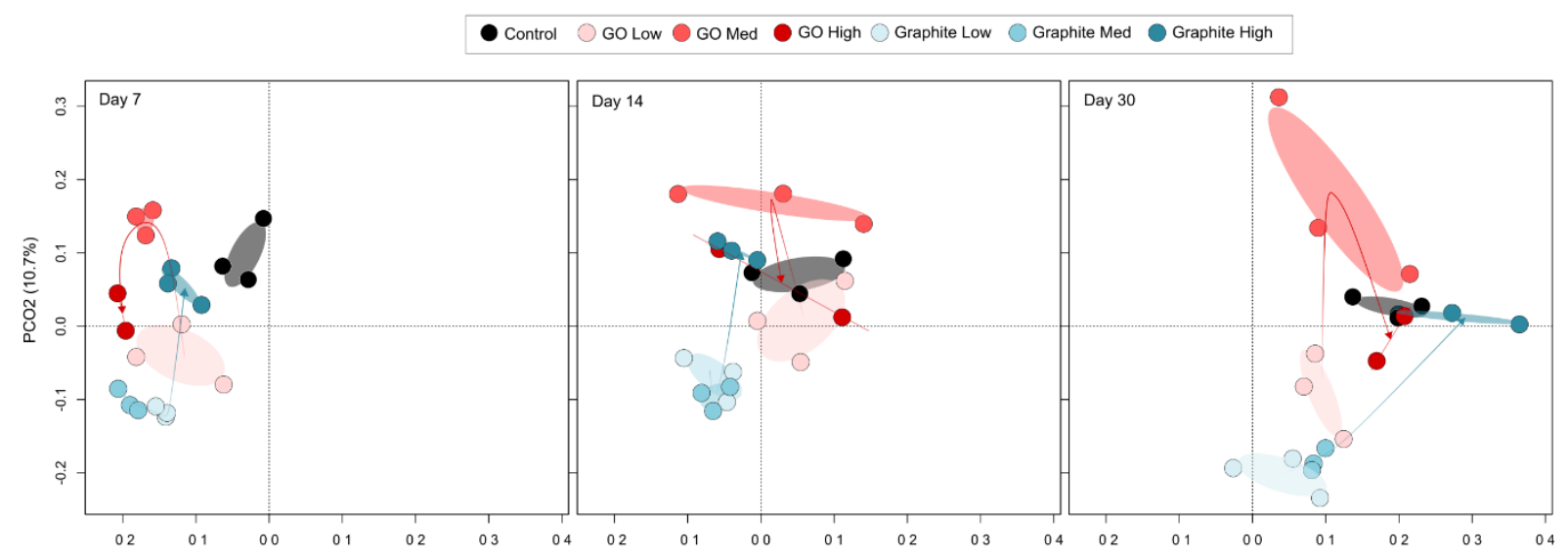

Fig. 2 Principal coordinate analysis (PCoA) ordination illustrating differences in the composition of bacterial communities in control, and graphite and GO amended soils over time. The ellipses represent standard deviations. The three panels, representing different time points, derive from a single ordination that included all samples. Each time point is shown separately to avoid overcluttering. The arrows for each treatment move from low, through medium, to high dose. They highlight that there was not a consistent direction of change in community composition with increasing GO or graphite dose. GO and graphite doses correspond to $1 \mathrm{ng}, 1 \mu \mathrm{g}$ and $1 \mathrm{mg} \mathrm{kg}^{-1}$ soil. 
bioRxiv preprint doi: https://doi.org/10.1101/530485; this version posted March 12, 2019. The copyright holder for this preprint (which was not certified by peer review) is the author/funder. All rights reserved. No reuse allowed without permission.

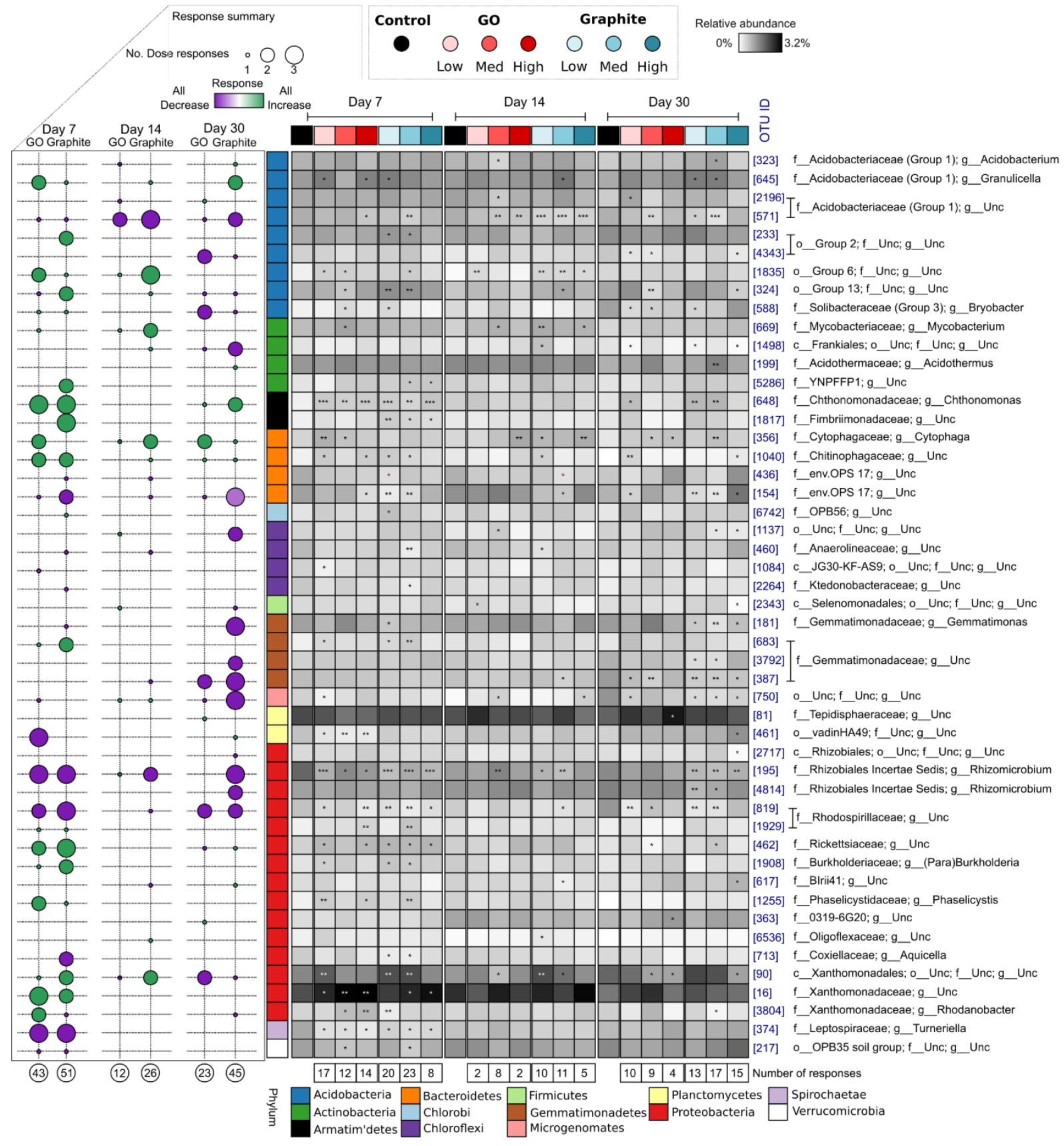

Fig. 3 Heatmap of the relative abundances of 49 bacterial OTUs that differed significantly from the control in at least one treatment combination. The asterisks highlight which treatments differ significantly from the controls on each day $\left(P<0.05^{*}, P<0.01^{* *}, P<0.001^{* * *}\right)$. Each column 
of the heatmap represents the mean relative abundance of each treatment $(n=3)$. The bubble-plot on the left summarizes the number (circle size) of GO or graphite doses that an OTU responded to relative to the controls, and of these how many manifested as increases or decreases in relative abundance (circle color). The numbers below the bubble plot and heatmap show the total numbers of significant responses to a particular treatment relative to the control within the same day. The OTU IDs are consistent throughout the manuscript. The phylum of each OTU is indicated by the colors on the left of the heatmap and the affiliations associated with each color are shown at the bottom. GO and graphite doses correspond to $1 \mathrm{ng}, 1 \mu \mathrm{g}$ and $1 \mathrm{mg} \mathrm{kg}^{-1} \mathrm{soil}^{\text {. }}$ 


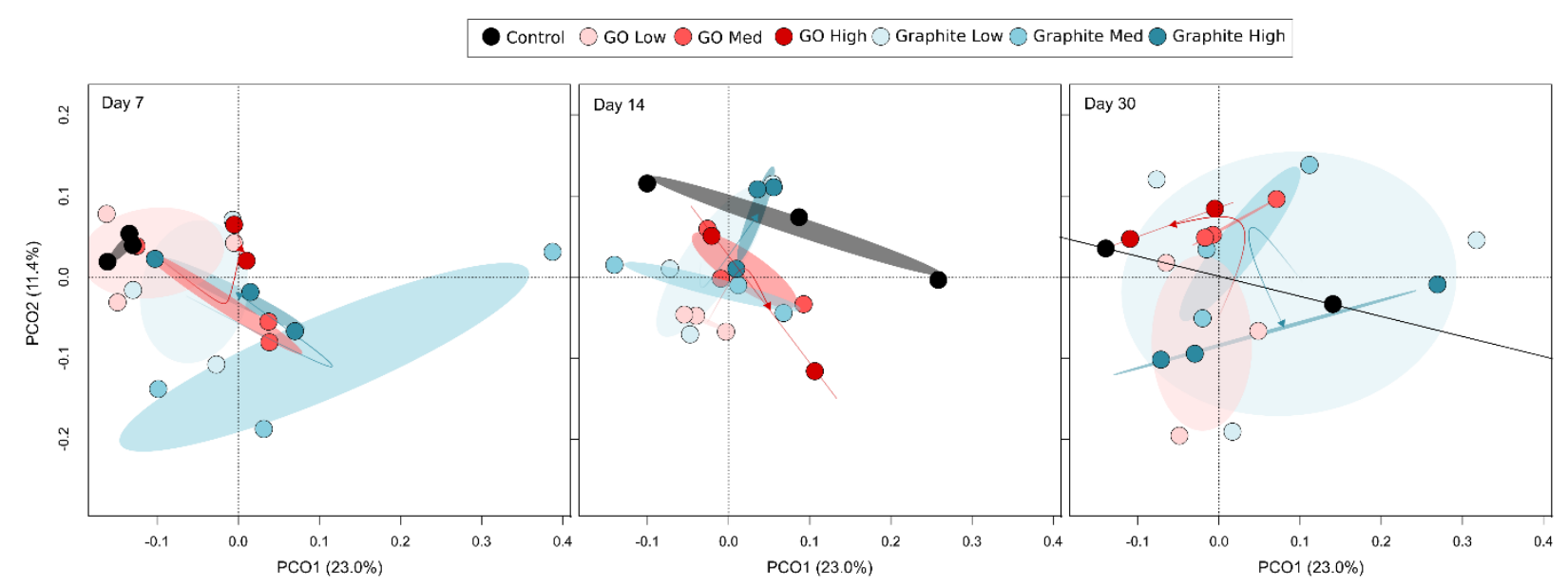

Fig. 4 Principal coordinate analysis (PCoA) ordination illustrating differences in the composition of fungal communities in control, and graphite and GO amended soils over time. The ellipses represent standard deviations. The three panels, representing different time points, derive from a single ordination that included all samples. Each time point is shown separately to avoid overcluttering. On day 30 , one of the control samples $(\mathrm{PCO} 1=1.2, \mathrm{PCO} 2=-0.07)$ is beyond the plotted area. The arrows for each treatment move from low, through medium, to high dose. They highlight that there was not a consistent direction of change in community composition with increasing GO or graphite dose. GO and graphite doses correspond to $1 \mathrm{ng}, 1 \mu \mathrm{g}$ and $1 \mathrm{mg} \mathrm{kg}^{-1}$ soil. 


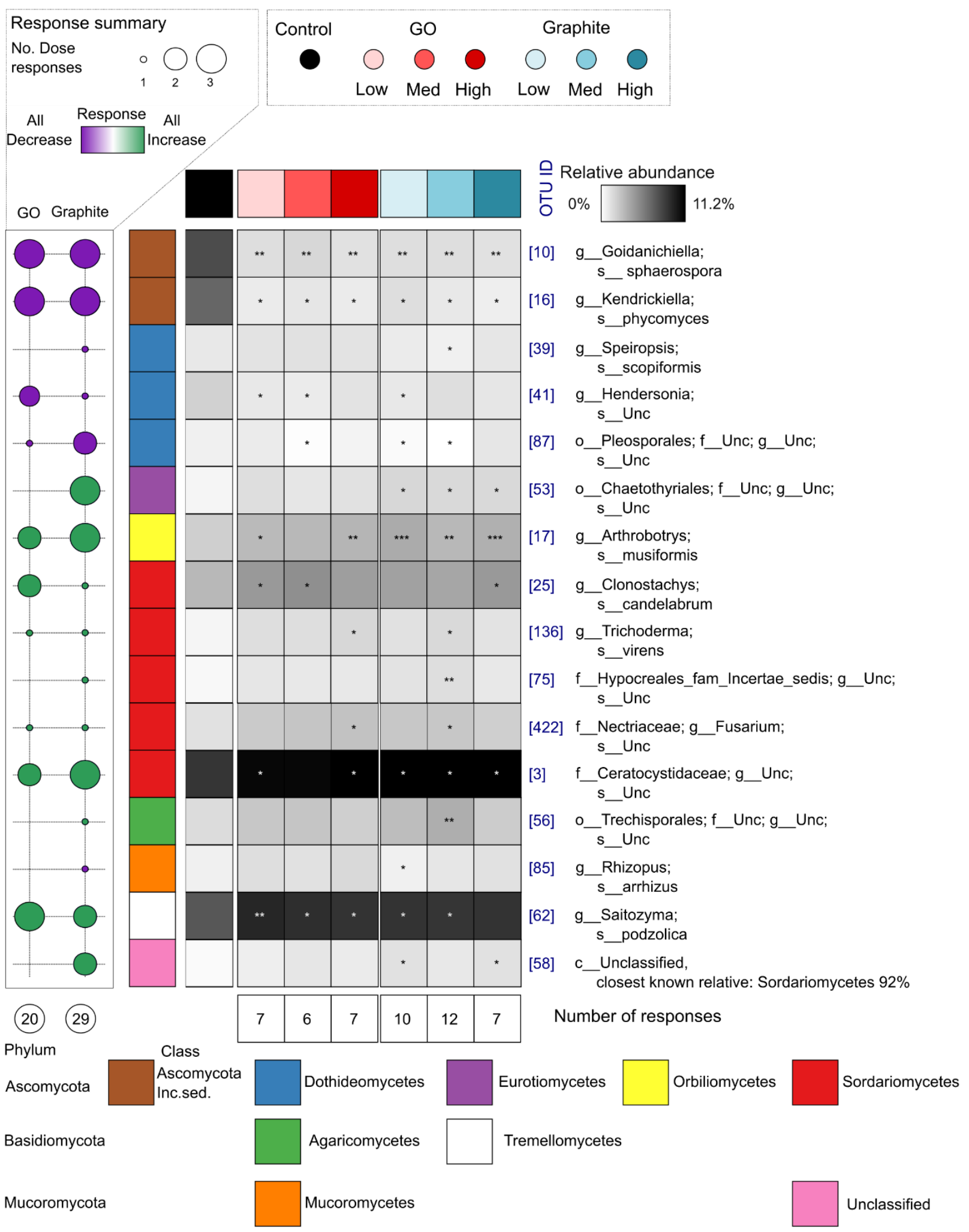


Fig. 5 Heatmap of the relative abundances of 16 fungal OTUs that differed significantly from the control in at least one treatment combination. The asterisks highlight which treatments differ significantly from the controls on each day $\left(P<0.05^{*}, P<0.01 * *, P<0.001 * * *\right)$. Each column of the heatmap represents the mean relative abundance of each treatment $(n=9)$. The bubble-plot on the left summarizes the number (circle size) of GO or graphite doses that an OTU responded to relative to the controls, and of these how many manifested as increases or decreases in relative abundance (circle color). The numbers below the bubble plot and heatmap show the total numbers of significant responses to a particular treatment relative to the control within the same day. The OTU IDs are consistent throughout the manuscript. The phylum and class of each OTU is indicated by the colors on the left of the heatmap and the affiliations associated with each color are shown at the bottom. GO and graphite doses correspond to $1 \mathrm{ng}, 1 \mu \mathrm{g}$ and $1 \mathrm{mg} \mathrm{kg}^{-1} \mathrm{soil}_{\text {. }}$ 
Table 1: Summary of multivariate GLM post-hoc results computed using mvabund highlighting difference in bacterial community composition between treatments relative to the controls within each time point.

\begin{tabular}{ll}
\hline \multicolumn{1}{c}{ Test } & $P$ value \\
\hline Day 7 GO Low & $<0.001^{* * *}$ \\
Day 7 GO Medium & $<0.001^{* * *}$ \\
Day 7 GO High & $<0.001^{* * *}$ \\
Day 7 Graphite Low & $<0.001^{* * *}$ \\
Day 7 Graphite Medium & $<0.001^{* * *}$ \\
Day 7 Graphite High & $<0.001^{* * *}$ \\
& \\
Day 14 GO Low & 0.140 \\
Day 14 GO Medium & $0.003^{* *}$ \\
Day 14 GO High & $0.020^{*}$ \\
Day 14 Graphite Low & $<0.001^{* * *}$ \\
Day 14 Graphite Medium & $<0.001^{* * *}$ \\
Day 14 Graphite High & $0.006^{* *}$ \\
& \\
Day 30 GO Low & $0.001^{* *}$ \\
Day 30 GO Medium & $<0.001^{* * *}$ \\
Day 30 GO High & $0.021^{*}$ \\
Day 30 Graphite Low & $<0.001^{* * *}$ \\
Day 30 Graphite Medium & $<0.001^{* * *}$ \\
Day 30 Graphite High & $0.001^{* *}$ \\
\hline
\end{tabular}


Table 2: Summary of multivariate GLM post-hoc results computed using mvabund highlighting difference in fungal community composition between treatments relative to the controls.

\begin{tabular}{|c|c|}
\hline Test & $P$ value \\
\hline GO Low & $0.001 * *$ \\
\hline GO Medium & $0.005^{* *}$ \\
\hline GO High & $0.005^{* *}$ \\
\hline Graphite Low & $0.002 * *$ \\
\hline Graphite Medium & $0.001 * *$ \\
\hline Graphite High & $0.001 * *$ \\
\hline
\end{tabular}

